Goal of the work: foal of our work is study of prevalence of food allergies and risk factors in children's population in selected populations of Tbilisi, Batumi and Kutaisi-Tskaltubo

Materials and methods Studied population includes 2655 children (2010-2013) from 1-month to 14-year age. 1359 of them were girls and 1296 - boys (I group: children from 1 month to 6 years and II group - from 6 years to 14 years). At the first stage of epidemiological study, screening of 2665 children was conducted by means of the initial questionnaire filled in directly at a time of interviewing of the parents. Identification of the factors of causal significance was provided based on anamnesis data, comparison of general serum and specific $\operatorname{IgE}$ and in vivo allergologic diagnostics (prick-tests). Obtained data were statistically processes by means of SPSS/V12.5 software (Statistical Package for Social Sciences).

Results Epidemiological studies showed that prevalence of food allergies in children's population (7.5\% - Tbilisi; 6.2\% - KutaisiTskaltubo; 4.3\% - Adjara) was 18.04\%. Average total IgE, im both cases, was 3-5 times higher than normal value and no statistically reliable difference between the groups was found $(\mathrm{p}>$ 0.05 ). Only 3.9 of children with food allergies had IgE within normal limits. High frequency of late diagnostics was established $(\mathrm{p}<0.001)$.

Conclusion Thus, according to the obtained data, share of the manageable risk factors is high and this could provide basis for development of targeted and effective prevention measures for the children's population. Food allergy is complex and versatile process requiring further study.

\section{P0-0992 SOME BONE MARKERS AND SERUM VITAMIN D IN EPILEPTIC CHILDREN ON EPILEPTIC DRUGS}

${ }^{1}$ E Abdel Hamid, ${ }^{1} \mathrm{H}$ Elnady, ${ }^{1}$ I Alameey, ${ }^{2} \mathrm{M}$ Girgis, ${ }^{3} \mathrm{LS}$ Sherif, ${ }^{4} \mathrm{I}$ Helwa. ${ }^{1}$ Child Health Department, National Research Center, Cairo, Egypt; ${ }^{2}$ Pediatric Department, Cairo University, Cairo, Egypt; ${ }^{3}$ Child Health Department, National Research Center, Cairo, Egypt; ${ }^{4}$ Immunogenetic Department, National Research Center, Cairo, Egypt

\subsection{6/archdischild-2014-307384.1609}

Aim to estimate serum vitamin D level and some biochemical markers of bone turnover in Egyptian children with epilepsy on antiepileptic drugs.

Methods Case control cross sectional study was conducted on thirty children with epilepsy (19 males, and 11 females) on anticonvulsant therapy, divided into two subgroups according to mode of therapy; 15 under polytherapy (group I) 15 under monotherapy (group II). Twenty apparently healthy children were recruited as control group.

Results Epileptic patients on polytherapy had highly significant low serum 25-hydroxy vitamin D level compared to those on monotherapy $(\mathrm{p}<0.001)$ with highly significant differences between patients versus controls $(\mathrm{p}<0.001)$. Over two third of patients $80 \%(24 / 30)$ had low serum 25-OHD levels; $26.67 \%$ $(8 / 30)$ had 25-hydroxyl vitamin D levels less than $20 \mathrm{ng} / \mathrm{mL}$, and $53.33 \%(16 / 30)$ patients had 25-hydroxy vitamin D levels between 21 and less than $32 \mathrm{ng} / \mathrm{ml}$. Differences between the cutoff categories were highly statistically significant for patients versus controls ( $p<0.001)$, and among the polytherapy versus monotherapy subgroups ( $\mathrm{p}<0.001$ ). Patients on polytherapy showed highly significant lower level of 25-hydroxyvitamin D compared to those on valoproate alone, or carbamazepine alone $(\mathrm{F}=32.345, \mathrm{p}<0.001)$ by ANOVA.
Conclusion Results revealed high risk of vitamin D deficiency in epileptic children on antiepileptic drugs especially those under long term polytherapy. Alterations of biochemical markers of bone formation suggest an accelerated skeletal turnover. Routine monitoring of serum 25 - hydroxy vitamin D is recommended.

\section{PO-0993 EPIDEMIOLOGY OF DRUG OVERDOSE/POISONING IN PAEDIATRIC POPULATION IN A SINGLE CENTRE OVER 2 YEAR PERIOD}

D Rallis, A Stefanopoulos, A Petropoulos, V Karakosta, K Angelopoulos, E Botsa, I Orfanou. Paediatrics, Aghia Sophia Children's Hospital, Athens, Greece

\subsection{6/archdischild-2014-307384.1610}

Bagkround Unintentional ingestion of toxic substances (cigarette), drug overdose and mucosal injuries due to corrosive liquate (house cleaners, batteries) remains common paediatric problem, with estimated annual admissions 94/100,000 population.

Methods We retrospectively recorded all patients admitted due to drug overdose-toxic/corrosive substance ingestion to the $3 \mathrm{rd}$ Department of First Paediatrics, of the National University of Athens Greece, from 3/2012-3/2014.

Results In total 190 patients were recorded, with mean age 41 \pm 39 months. The study population was further divided into 4 age-groups: babies (0-12 months), toddlers (1-3 years), prepubertal children (4-8 years) and teenagers $(>9$ years). Males were predominant in younger age $(68 \%, 57 \%$ and $62 \%$ in group 1,2 and 3 respectively), while females were predominant during pubertal age $(75 \%),(p<0.05)$. Babies' main hospitalising reason was cigarette ingestion (55\%). Toddlers were equally admitted due to cigarette (34\%), corrosive liquate ingestion $(22 \%)$ or drug overdose (33\%). Drug overdose was mainly recorded in prepubertal children (60\%), while teenagers mainly suffered by drug $(45 \%)$ or alcohol overdose $(40 \%)$, $(\mathrm{p}<0.001)$. Regarding the duration of the hospitalisation, younger patients (groups 1, 2 and 3 ) discharged in $<24 \mathrm{~h}$ (91\%, 80\% and $75 \%$ respectively), $55 \%$ of teenagers however had to stay for $>24 \mathrm{~h}(\mathrm{p}<0.001)$. No differences were recorded regarding the socialeconomical/ educational status of the families, notable is however that $27 \%$ of the patients were self-discharged, unlike doctors' suggestions. Conclusion Young males with unintentional cigarette ingestion/ drug overdose, or teenage females with alcohol/drug overdose comprise the profile of our typical patient. Despite the initial family stress, $25 \%$ of the patients are shortly after admission self-discharged.

\section{PO-0993a FATTY ACID BINDING PROTEINS AS AN UPRISING NON INVASIVE PREDICTOR OF GUT WALL INTEGRITY LOSS IN VIRAL GASTROENTERITIS}

${ }^{1} \mathrm{MT}$ Saleh, ${ }^{1} \mathrm{HG}$ Elnady, ${ }^{1} \mathrm{LS}$ Sherif, ${ }^{1} \mathrm{MM}$ Youssef, ${ }^{2} \mathrm{Al}$ El Shafie, ${ }^{1} \mathrm{IR}$ El-Alameey, ${ }^{3} \mathrm{NM}$ Kholoussi, ${ }^{3} \mathrm{I}$ Helwa, ${ }^{3} \mathrm{H}$ Abdel Raouf, ${ }^{4} \mathrm{AN}$ El-Taweel. ${ }^{1}$ Child Health, National Research Center, Cairo, Egypt; ${ }^{2}$ Health Radiation Research, National Center for Radiation Research and Technology, Cairo, Egypt; ${ }^{3} /$ mmunogenetics, National Research Center, Cairo, Egypt; ${ }^{4}$ Water Pollution, National Research Center, Cairo, Egypt

\subsection{6/archdischild-2014-307384.1611}

Background and aim Serum intestinal fatty acid binding proteins (I-FABPs) are cytosolic proteins mainly expressed in the intestinal villi which are affected early in viral gastroenteritis (GE) 
pathphysiology. So it can be used as an early and sensitive marker for the evaluation of gut wall integrity loss in GE particularly rotavirus gastroenteritis (RV-GE) in Egypt.

Patients and methods This case-control cross-sectional study was conducted on 93 Egyptian cases who suffered from acute viral gastroenteritis. 28 healthy children matching in age were recruited as the control group. We collect all clinical data concerning disease manifestations and severity criteria.

Serum I-FABPs were measured using the Enzyme linked Immune Sorbent Assay (ELISA) technique. Viral detection and typing was done by Polymerase Chain Reaction (PCR) for adenovirus, and by Reverse transcriptase PCR (RT-PCR) for rotavirus, astrovirus and norovirus.

Results Results of this work revealed that serum I-FABPs levels were generally higher in the study group cases compared to the control group $(1026.4 \pm 494.4 \mathrm{pg} / \mathrm{ml}$ versus $267.9 \pm 200.4 \mathrm{pg} /$ $\mathrm{ml}, \mathrm{P}<0.001)$. They were also significantly higher in the 46 RV-GE cases compared to other types. Furthermore, Serum IFABPs levels were significantly higher in severely dehydrated cases as compared to mildly dehydrated ones $(p=0.037)$. Serum I- FABPs levels were correlated significantly with those hospitalised $(\mathrm{r}=0.223, \mathrm{p}<0.05)$.

Conclusion Serum I-FABPs can be successfully used not only as early and sensitive predictor marker of gut wall integrity loss in viral GE (especially RV-GE) but also their levels can indicate case severity.

\section{Pulmonology/Allergy/Immunology/Asthma}

\section{PO-0994 LTRI IN PAEDIATRICS: ANALYSIS OF AN ANNUAL SURVEY}

S Accomando, G Ferrante, A Tricarico, P Alga, MS Lo Presti, V Scavone, F Ardolino, F Leone, $\mathrm{G}$ Corsello. Department of Sciences for Health Promotion and Mother and Infant Care "G. D'Alessandro", Pediatrics Section, Palermo, Italy

10.1136/archdischild-2014-307384.1612

Background and aims Lower tract respiratory infections (LTRI) are very frequent in paediatric population. We collected epidemiological, etiological and clinical data and correlated them to some variables.

Methods We performed an observational study of all children with a LTRI, admitted to our Paediatric Unit from February 2013 to January 2014. The population was grouped in three classes of ages $(0-2 \mathrm{yr}, 3-6 \mathrm{yr},>6 \mathrm{yr})$. All patients underwent to a questionnaire focused on exposition to protective and risk factors for respiratory diseases.

Results 83 children were included. We grouped them according to discharge diagnosis and analysed the distribution for ages, sex and season of onset. At the admission 18 patients had respiratory distress; $\mathrm{O} 2$ therapy was necessary for 11 of them, infusion therapy for 40, endovenous antibiotic therapy for 34 .

Swabs were positive in 1 case for Parainfluenza viruses and in 2 for S. Aureus; sierological tests were positive in 5 cases for

\begin{tabular}{lllllll}
\multicolumn{2}{l}{ Abstract P0-0994 Table 1} \\
\hline Diagnosis & N & Sex & $0-2 \mathrm{yr}$ & $3-6 \mathrm{yr}$ & $>6 \mathrm{yr}$ & Hospital day \\
Pneumonia & 56 (43 Typical-13 Atypical) & $35 \mathrm{M}-21 \mathrm{~F}$ & 17 & 17 & 22 & 6,28 \\
Bronchiolitis & 9 & $6 \mathrm{M}-3 \mathrm{~F}$ & all & & & 5,1 \\
Bronchitis & 9 & $6 \mathrm{M}-3 \mathrm{~F}$ & 2 & 4 & 3 & 7,6 \\
Wheezing & 9 & $5 \mathrm{M}-4 \mathrm{~F}$ & 1 & 5 & 3 & 3,4 \\
\hline
\end{tabular}

Mycoplasma Pneumoniae, in 2 for Chlamydia Pneumoniae, in 1 for ParvovirusB19, in 1 for Coxsackievirus. In 28 patients (33\%) exposition to passive smoke was observed, in 17 (20\%) to aeroallergens and in $33(40 \%)$ a personal or familiar story of atopy. Conclusions Our experience showed a higher prevalence of LTRI in males (63\%), in winter (35\%) and spring (33\%), without a difference between preschool and school age children.

\section{PO-0995 SWEAT TESTING SINCE THE INTRODUCTION OF NEWBORN SCREENING IN WEST MIDLANDS, UK}

${ }^{1}$ M Ahmed, ${ }^{2}$ T Reynolds. ${ }^{1}$ Paediatrics, Burton Hospitals NHS Foundation Trust, Burton on Trent, UK; ${ }^{2}$ Clinical Chemistry, Burton Hospitals NHS Foundation Trust, Burton on Trent, UK

\subsection{6/archdischild-2014-307384.1613}

Background Cystic Fibrosis (CF) is an autosomal recessive condition caused by gene mutation which affects sodium and chloride transport across the membrane of secretory epithelial cells. New born screening for CF was introduced in the West Midlands, UK in November 2006. 20\% of CF patients may present with meconium ileus. The majority of the remainder are expected to be picked through new-born screening. Sweat test remains the gold standard for the diagnosis of $\mathrm{CF}$ and is a critical component of +ve newborn CF screening protocol.

Aim To investigate the positive yield of sweat test at Queen's Hospital Burton Upon Trent (in patients with negative new born CF screen) since the introduction of new born CF screening.

Methods We retrospectively collected local data on all the sweat test results since the introduction of new born CF screening in the West Midlands.

Results Out of 129 sweat tests performed, only one case yielded positive result (born before new born CF screening). Another patient had a borderline test result which was subsequently repeated and found to be normal. Therefore, we effectively have no positive sweat test results so far since the screening commenced.

Conclusion Even though our data is encouraging and suggests increasing the threshold required for performing a sweat test (in individuals born after Nov 2006), this investigations should still be carried out in patients with high index of clinical suspicion as occasional cases will be missed despite universal new born $\mathrm{CF}$ screening programme.

\section{PO-0996 ELEVATED LEVELS OF INTERFERON-INDUCIBLE PROTEIN 10 (IP-10) IN PATIENTS WITH 22Q11.2 DELETION (DIGEORGE) SYNDROME}

${ }^{1} \mathrm{DM}$ Aresvik, ${ }^{2} \mathrm{~K}$ Lima, ${ }^{3} \mathrm{~T}$ Overland, ${ }^{4} \mathrm{TE}$ Mollnes, ${ }^{5} \mathrm{TG}$ Abrahamsen. ${ }^{1}$ Department of Paediatric Research, Oslo University Hospital, Oslo, Norway; ${ }^{2}$ Division of Medicine, Akershus University Hospital, Lorenskog, Norway; ${ }^{3}$ Women and Children's Division, Oslo University Hospital, Oslo, Norway; ${ }^{4}$ Department of Immunology, Oslo University Hospital and University of Oslo, Oslo, Norway; ${ }^{5}$ Women and Children's Division, Oslo University Hospital and University of Oslo, Oslo, Norway

\subsection{6/archdischild-2014-307384.1614}

Background and aim The 22q11.2 deletion syndrome (DS), also known as DiGeorge syndrome, is a genetic disorder with an estimated incidence of 1 in 4000 births. These patients may suffer from disorders of many organ systems, but cardiac malformations, thymic hypoplasia/aplasia, hypoparathyroidism, cleft palate and psychiatric disorders are most frequent. In addition, the 